\title{
Overexpression of the antiapoptotic protein A1 promotes the survival of double positive thymocytes awaiting positive selection
}

\author{
C Verschelde ${ }^{1}$, D Michonneau ${ }^{1}$, M-C Trescol-Biemont ${ }^{1}$, \\ I Berberich ${ }^{2}$, A Schimpl ${ }^{2}$ and N Bonnefoy-Berard ${ }^{\star, 1}$ \\ ${ }^{1}$ Laboratory of homéostasie lymphocytaire, INSERM U503, IFR Biosciences \\ Lyon-Gerland, 21 avenue Tony Garnier, 69007 Lyon, France \\ 2 Institute for Virology and Immunobiology, University of Würzburg, Versbacher \\ Str. 7, 97078 Wüerzburg, Germany \\ * Corresponding author: N Bonnefoy-Berard, INSERM U503, IFR Biosciences \\ Lyon-Gerland, 21 avenue Tony Garnier, 69007 Lyon, France. \\ Tel: + 33-437-28 23-72; Fax: + 33-437-28-23-41; \\ E-mail: bonnefoy@cervi-lyon.inserm.fr
}

Received 22.7.05; revised 12.9.05; accepted 07.10.05; published online 18.11.05 Edited by A Strasser

\begin{abstract}
As it has been shown for Mcl-1, Bcl-xl and Bcl-2, proteins of the Bcl-2 family play a crucial role during T-cell development in the thymus. We here show that the expression of the antiapoptotic gene A1 is specifically enhanced at the DN3/ DN4 transition and in DP thymocytes that have been positively selected suggesting that A1 expression might be considered as a transcriptional signature of thymocytes that have received pre-TCR or TCR survival signal. Furthermore, we observed that A1-a overexpression in recombination activation gene 1-deficient mice transgenic for the major histocompatibillity complex class I-restricted F5 TCR enhances cell survival of DP thymocytes and permits accumulation of DP cells awaiting positive selection. However, A1-a overexpression has no effect on negative selection. Therefore, our results suggest that $A 1$ plays a specialized role in allowing survival of DP thymocytes and that its role can be distinguished from that of $\mathrm{Mcl}-1, \mathrm{Bcl}-\mathrm{xl}$ and Bcl-2.

Cell Death and Differentiation (2006) 13, 1213-1221.

doi:10.1038/sj.cdd.4401814; published online 18 November 2005
\end{abstract}

Keywords: A1; Bcl-2; DP thymocytes; positive selection

Abbreviations: BrdU, 5-bromo-2'-deoxyuridine; DP, double positive; DN, double negative; PBS, phosphate buffer saline; $\mathrm{SP}$, single positive; TCR, T-cell receptor; Tg, transgenic

\section{Introduction}

Apoptosis plays a crucial role in the development and maintenance of an efficient immune system. The development of $\mathrm{T}$ cells in the thymus is characterized by the production of large numbers of immature thymocytes that undergo several steps of selection. Immature $\mathrm{CD} 4^{-} \mathrm{CD} 8^{-}$ double negative (DN) cells, that are also T-cell receptor (TCR) negative, can be subdivided on the basis of CD44 and CD25 expression into four sequential developmental stages: DN1 $\left(\mathrm{CD} 44^{+} \mathrm{CD} 25^{-}\right)$, DN2 $\left(\mathrm{CD} 44^{+} \mathrm{CD} 25^{+}\right)$, DN3 $\left(\mathrm{CD} 44^{-} \mathrm{CD} 25^{+}\right)$and $\mathrm{DN} 4\left(\mathrm{CD} 44^{-} \mathrm{CD} 25^{-}\right)$. For the cells committed in the $\alpha \beta$ T-cell lineage, TCR $\beta$ rearrangement occurs at the DN3 stage. A functional TCR $\beta$ chain resulting from successful rearrangement assembles with the preTCR $\alpha$-chain $(p T \alpha)$ and CD3 chains to form the pre-TCR complex. Pre-TCR signals allow survival and further differentiation to the DN4 stage. Thus, the transition from DN3 stage to DN4 represents the first checkpoint in the development of T cells and is referred to as $\beta$ selection. ${ }^{1}$ This step allows survival and further differentiation of cells that had produced a functional TCR $\beta$ chain. After the DN4 stage, immature thymocytes upregulate CD8 and CD4 expression, becoming $\mathrm{CD} 4^{+} \mathrm{CD}^{+}$double positive (DP) cells. The resulting DP population has an intrinsic life span of 3-4 days, during which they undergo multiple rounds of TCR $\alpha$ rearrangements to maximize the chances of forming a functional TCR $\alpha \beta$ heterodimer. ${ }^{2}$ However, a great majority of DP thymocytes fail to form a TCR that interacts productively with self peptide/ $\mathrm{MHC}$ complexes and die by neglect as a result of the failure to receive an appropriate signal for the positive selection. In addition, negative selection leads to the deletion of DP thymocytes that express high affinity, potentially autoreactive, receptors. Thus, these three selection steps allow the differentiation of T cells, bearing functional TCR, that egress the thymus.

The life span of DP thymocytes depends on proper signals mediated first by the pre-TCR and then by the TCR. These signals ultimately regulate the expression of $\mathrm{Bcl}-2$ family prosurvival members, which control susceptibility of DP cells to apoptosis. Indeed, $\mathrm{Bcl}-\mathrm{xl}$ is highly expressed in DP cells and is thought to promote their survival as its deletion dramatically shortens their life span. ${ }^{3} \mathrm{Bcl}-2$ is initially expressed at low levels in these cells, but is upregulated when DP cells are positively selected. ${ }^{4-6}$ In addition, forced expression of $\mathrm{BCl}-2$ greatly increases the survival of DP cells in the absence of positive selection. ${ }^{7-9}$ So far there is no evidence for a specific role of $\mathrm{Mcl}-1$ at the DP stage, the loss of $\mathrm{Mcl}-1$ was demonstrated to increase apoptosis of DN2 cells and to arrest thymocyte development at the DN3 stage. ${ }^{10}$

In a previous study, we demonstrated that $\mathrm{A} 1$, a more recently identified pro-survival gene of the Bcl-2 family, is upregulated following TCR but not $\gamma \mathrm{C}$-cytokine receptor engagement in mature $\mathrm{T}$ lymphocytes. ${ }^{11}$ More recently, Mandal et al. ${ }^{12}$ identified $\mathrm{A} 1$ as a pre-TCR-induced gene and showed that retroviral expression of A1 allows survival and differenciation of pre-TCR ${ }^{-}$thymocytes from $\mathrm{Rag}^{-/-}$ mice. In the current report, we sought to determine the role of A1 on the selection of DP thymocytes. We show that the prosurvival gene $\mathrm{A} 1$ is upregulated in DP cells that have been 
positively selected and that overexpression of A1 in RagF5 TCR transgenic $(\mathrm{Tg})$ mice enhances cell survival of DP thymocytes and permits the accumulation of DP cells awaiting for positive selection. However, A1 overexpression has no effect on negative selection.

\section{Results}

\section{A1 expression in thymocytes}

We and others recently identified $\mathrm{A} 1$ as an antiapoptotic gene of the Bcl-2 family that is regulated by signals initiated from TCR in mature peripheral T cells ${ }^{11}$ and pre-TCR in thymocytes. ${ }^{12}$ As both pre-TCR and TCR signals are essential for the development of $T$ cells in the thymus, those data suggested that $A 1$ may play a major role in regulating $T$-cell survival during T-cell development. Real-time RT-PCR analysis using CDNA isolated from FACS-sorted DN3, DN4, DP as well as CD8 and CD4 single positive (SP) thymocytes from $\mathrm{C57BL} / 10$ mice, reinforced this hypothesis. Real-time RT-PCR has been done using primers that coamplify all the three functional isoforms $A 1-a, A 1-b$ and $A 1-d$. As shown in Figure 1, we observed that DN3 thymocytes expressed very low level of A1 mRNA. However, its expression was strongly upregulated (x9) in the DN4 subpopulation. In agreement with previous results ${ }^{12}$ such upregulation at the DN3/DN4 transition is specific for A1 mRNA since expression of $\mathrm{Bcl}-\mathrm{xl}, \mathrm{Bcl}-2$ and $\mathrm{Mcl}-1$ are either marginally enhanced or downregulated. A1 expression is downregulated in DP thymocytes and then reexpressed at high levels in both CD8 and CD4 SP thymocytes. These data suggest that $A 1$ expression might be considered as a transcriptional signature of thymocyte subsets that had received pre-TCR or TCR survival signals.

\section{Increased thymic cellularity in A1-a transgenic mice}

To assess the role of $\mathrm{A} 1$ during $\mathrm{T}$ cell development we developed A1-a Tg mice, using the human CD2 promoter to direct overexpression of A1-a in T cells. RagF5-A1 mice were generated by serial crossing of RagF5 with $\mathrm{TgA} 1$ mice. The TCR F 5 is formed by combination of $\mathrm{V} \alpha 4$ and $\mathrm{V} \beta 11$ members of the $\alpha$ and $\beta$ chain variable-region gene families, and recognizes a peptide of the influenza virus $A / N T / 60 / 68$ nucleoprotein in the context of $\mathrm{H}_{2} \mathrm{D}^{\mathrm{b}}{ }^{13}$ Therefore, expression of the F5 TCR skews postive selection towards the CD8 compartment. ${ }^{14}$ We observed that the $A 1-a$ transgene is efficiently expressed in DN and DP thymocytes, but not in CD8 SP thymocytes or mature peripheral CD8 T cells in spleen or lymph nodes (data not shown). Real-time RT-PCR analysis using primers that coamplify all the three functional isoforms and the transgene, revealed, respectively, 2.8 and four times more A1 expression in DN and DP thymocytes from RagF5A1 when compared to RagF5 control mice (Figure 2a).

We next analyzed whether this overexpression of $A 1$ in $D N$ and DP thymocytes is associated with an increased thymic cellularity in RagF5-A1 mice. Thymuses from either RagF5A1 or RagF5 control mice were collected from 1 day to more than 12 weeks after birth and total cell numbers as well as relative proportion of each thymocyte subpopulation were determined. We observed an increase in thymocyte numbers as soon as 1 week after birth in RagF5-A1 Tg mice, with a maximal thymic cellularity at 6 weeks in both control and
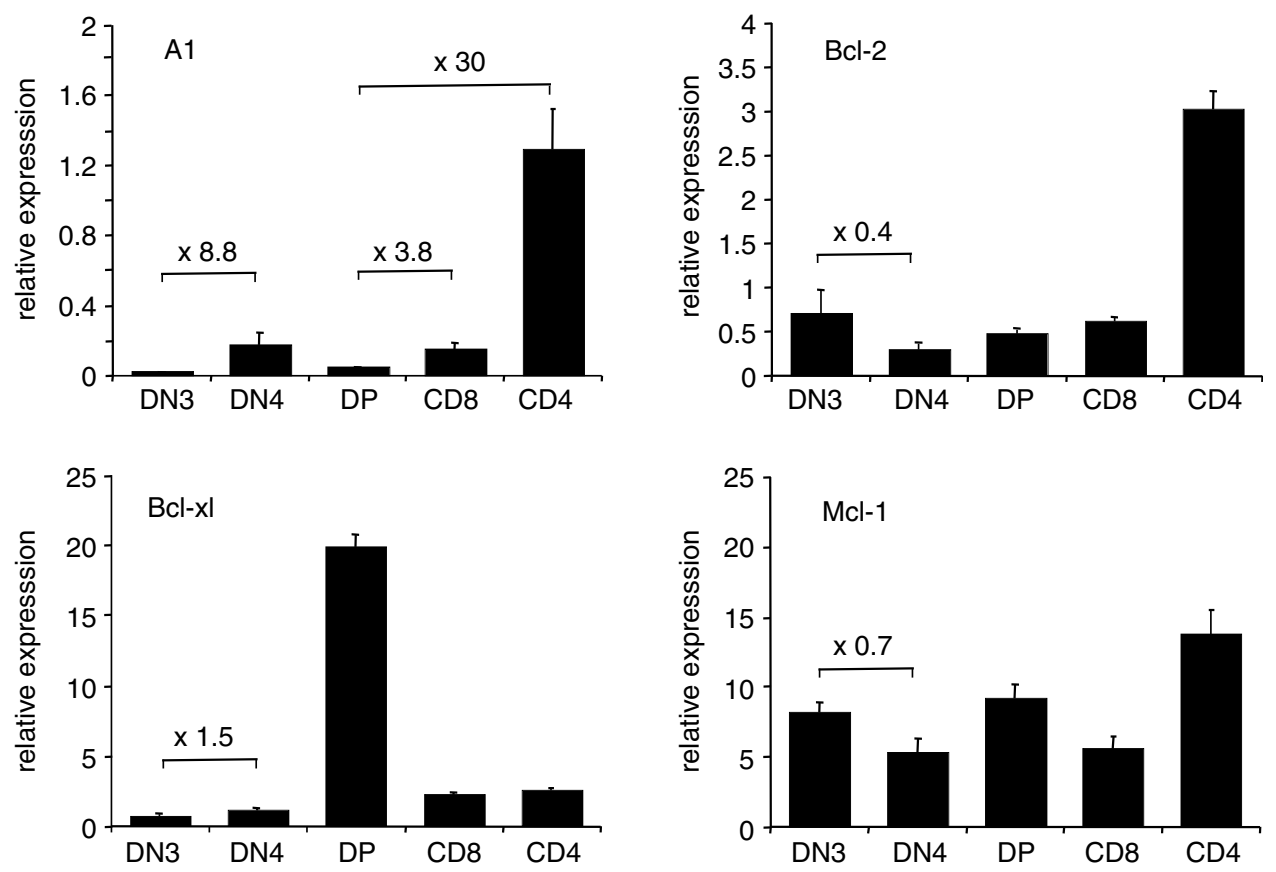

Figure 1 Expression of $\mathrm{A} 1, \mathrm{Bcl}-2, \mathrm{Bcl}-\mathrm{xl}$ and Mcl-1 mRNA in thymocytes subpopulations. Thymocyte subpopulations from C57BL/10 mice were FACS-sorted and analyzed for A1, Bcl-2, Bcl-xl and Mcl-1 mRNA content by real-time RT-PCR as described in Materials and methods. Results are expressed as the mean \pm S.E.M. of three independent experiments 

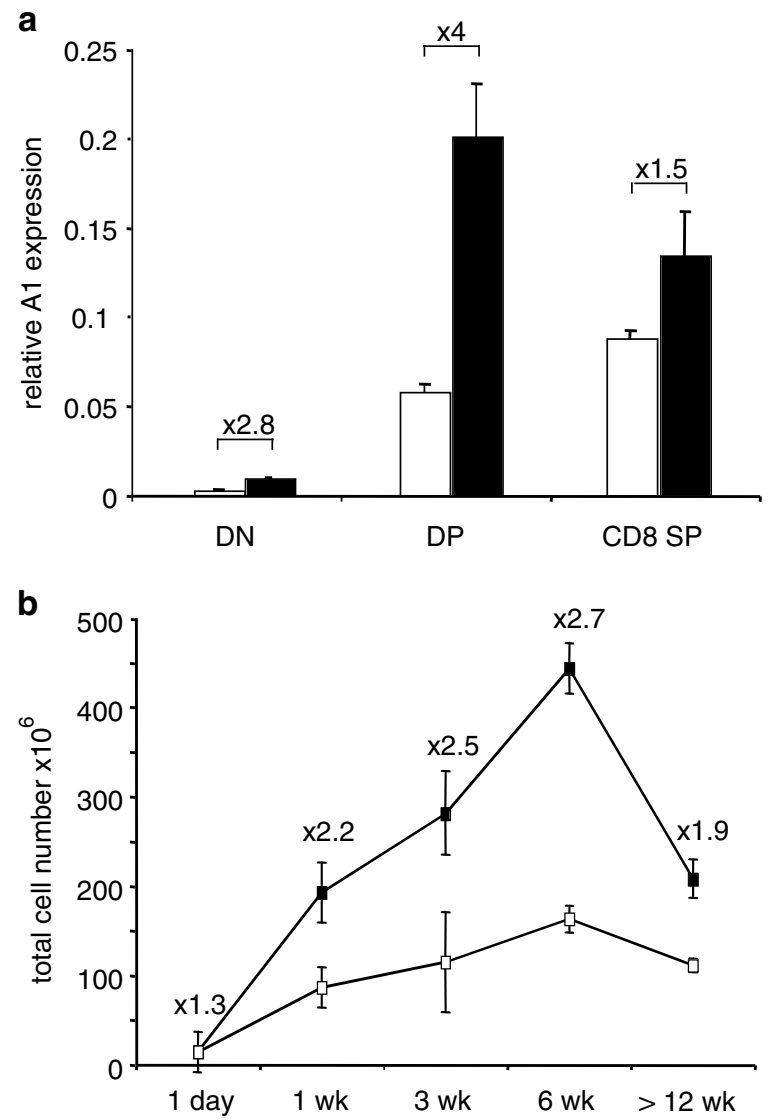

C

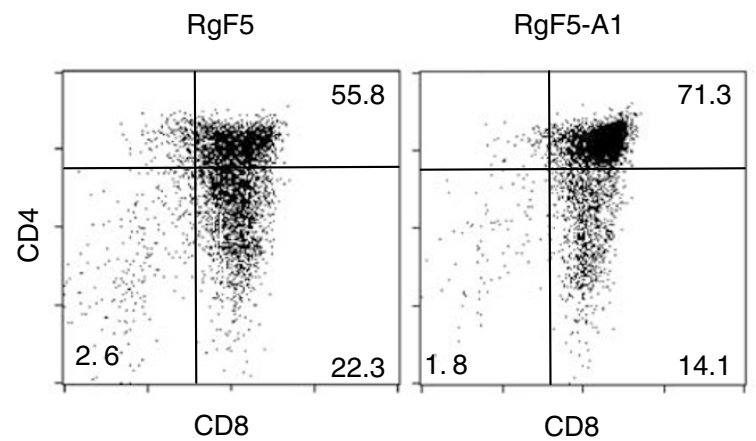

d

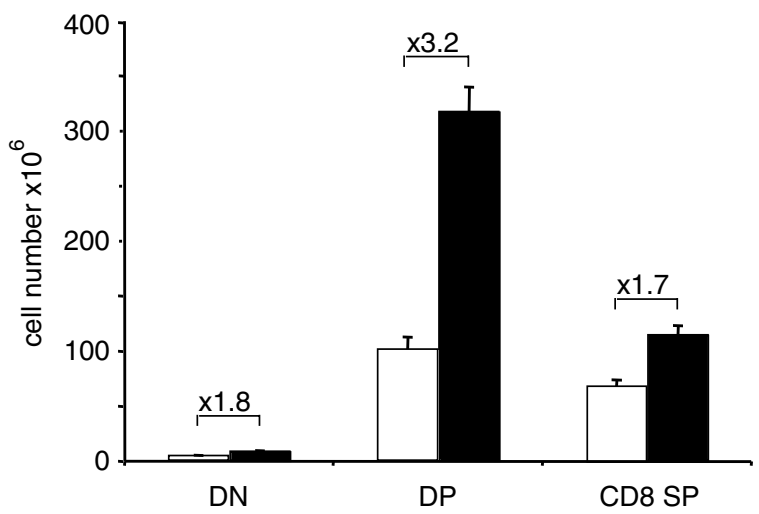

TgA1. RagF5-A1 Tg mice have an average of 2.7 times more cells than control mice at 6 weeks of age (Figure $2 b$ ). Increased thymocyte number is associated with a difference in the relative proportion of thymocyte subsets (Figure 2c) leading to a stronger accumulation of DP cells $(\times 3.2)$ than DN $(\times 1.8)$ or CD8 SP cells $(\times 1.6)$ in RagF5-A1 Tg mice (Figure 2d). Our results indicate that a three- to four-fold overexpression of the A1 gene in thymocytes is sufficient to significantly increase the number of these cells in developing thymus.

\section{A1 overexpression increases the survival of DP thymocytes}

Thymocytes isolated from mice that overexpress antiapoptotic proteins of the Bcl-2 family such as Bcl-2 itself or Bcl-xl show reduced spontaneous apoptosis in culture as well as resistance to cell death induced by CD3-TCR engagment, glucocorticoids, ionizing radiation. ${ }^{15-18}$ We analyzed whether the accumulated DP thymocytes in RagF5-A1 Tg mice would also be more resistant to cell death. FACS-sorted DP thymocytes from either RagF5-A1 or RagF5 control mice were tested for their sensitivity to spontaneous apoptosis as well as glucocorticoid- and TCR-induced apoptosis. Whatever the stimulus tested, DP from RagF5-A1 mice survived better than control cells (Figure $3 a$ ). However the protective effect of $A 1$ overexpression remains lower than that reported for overexpression of $\mathrm{Bcl}-2$ and $\mathrm{Bcl}-\mathrm{xl} .{ }^{15-17}$ Increased numbers of thymocytes in RagF5-A1 may also result from a higher proliferation rate of thymocytes. We therefore examined thymocyte proliferation in vivo by injecting 5-bromo-2'deoxyuridine (BrdU) into RagF5-A1 and RagF5 control mice. At $5 \mathrm{~h}$ after injection, thymocytes were removed, stained with antibodies against CD4, CD8 and BrdU, and the percentage of cells in each subset that had incorporated BrdU was determined. Similar percentage of BrdUpositive cells, were observed in DN, DP and CD8 subsets from both RagF5-A1 and RagF5 control mice. These results suggest that accumulation of DP in RagF5-A1 is mainly due to an increased survival rather than an increased proliferation of thymocyte subsets.

\section{A1 overexpression permits the accumulation of non-positively selected DP thymocytes}

DP thymocytes are the target for both positive and negative selection two processes, that are regulated by TCR signaling. ${ }^{19}$ We previously identified $\mathrm{A} 1$ as a direct target of

Figure 2 Increased thymic cellularity in RagF5-A1 Tg mice. (a), Thymocyte subpopulations from RagF5 (white) and RagF5-A1 (black) Tg mice were FACSsorted and analyzed for A1 mRNA content by real-time RT-PCR as described in Materials and methods. Results are expressed as the mean \pm S.E.M. of three independent experiments. (b), Total thymocytes numbers from RagF5 (white square) and RagF5-A1 (black square) Tg mice were evaluated at the indicated age of mice. The results are the mean \pm S.E.M. of six to 20 mice in each group. (c), Thymocytes from 6-week-old RagF5 and RagF5-A1 were analyzed by flow cytometry for CD4 and CD8 expression. The percentage of DN, DP and CD8 SP are shown in the dot-plot. (d), The numbers of DN-, DP- and CD8 SP-cells from 6-week-old RagF5 (white) and RagF5-A1 (black) tg mice were calculated from the total number of cells from thymus and and the percentage of these cells. Results are expressed as the mean \pm S.E.M. of three independent experiments 
a

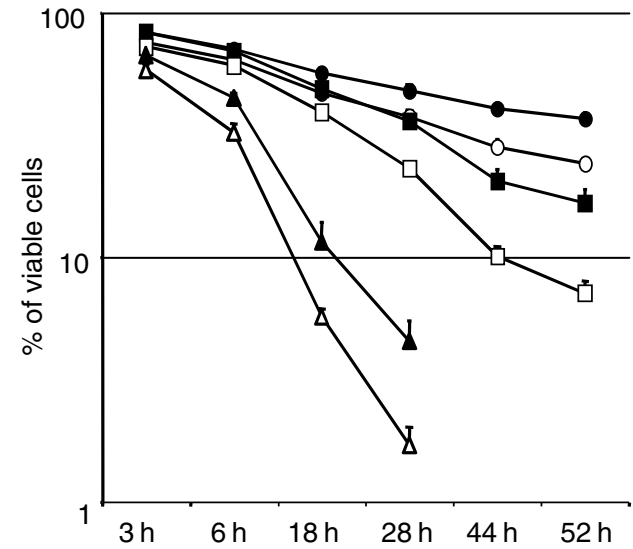

b

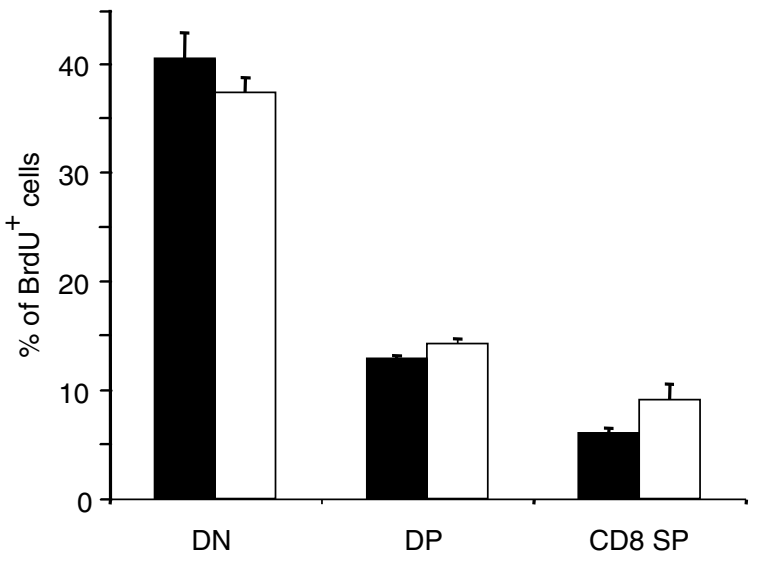

C

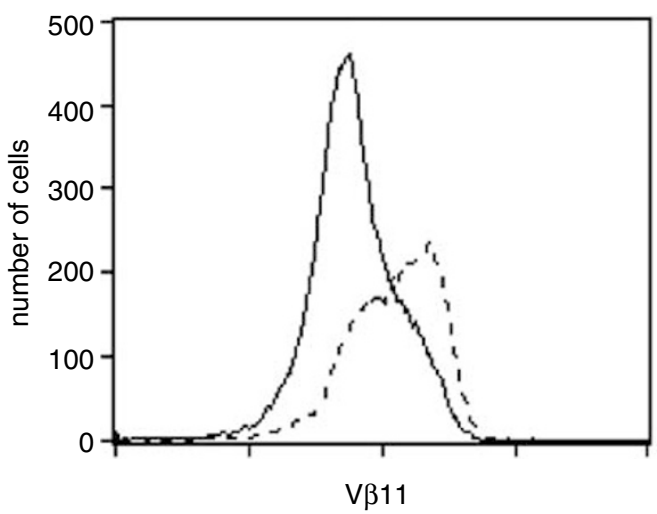

d

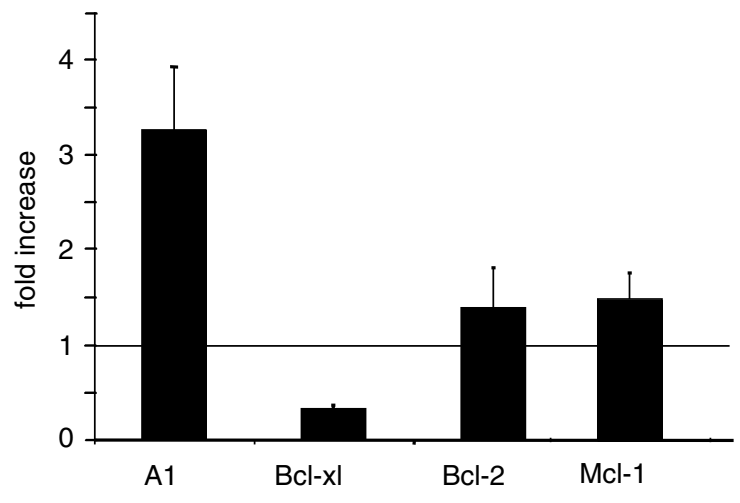

TCR-mediated signals in mature T cells, ${ }^{11}$ suggesting that $\mathrm{A} 1$ may play a major role in TCR-mediated survival. We therefore analyzed whether overexpression of $A 1$ would affect positive or negative selection in RagF5 mice.

TCR density on DP cells can be used to discriminate between cells that had or had not undergone positive selection. ${ }^{20}$ Such characteristic is applicable to RagF5 transgenic mice. Indeed it has been demonstrated that TCR expression in RagF5 mice is upregulated during the transition from DP through $\mathrm{CD} 4^{\mathrm{lo}} \mathrm{CD} 8^{\text {hi }}$ to $\mathrm{CD} 4^{-} \mathrm{CD} 8^{\text {hi }},{ }^{9}$ and that blockade of positive selection in mice lacking the $\mathrm{CD} 3 \gamma$ chain or in CD $3 \gamma$ - $\triangle$ ITAM mutant mice results in the accumulation DP thymocytes that express low level of F5 TCR. ${ }^{21}$ We therefore analyzed F5 TCR expression on DP cells from both RagF5-A1 and RagF5 control mice by flow cytometry with either antibodies against $\mathrm{V} \beta 11$, recognizing the F5 TCR- $\beta$ chain, or with specific pentamer. We observed that the percentage of $\mathrm{V} \beta 11^{\text {low }}$ expressing cells is highly increased in DP from RagF5-A1 mice when compared with RagF5 control mice (Figure $3 c$ ), suggesting an increased survival of nonpositively selected DP thymocytes in RagF5-A1 mice. Similar results were obtained following pentamer staining (data not shown). In parallel, A1 expression in FACS-sorted $\mathrm{V} \beta 11^{\text {low }}$ and $\mathrm{V} \beta 11^{\text {high }} \mathrm{DP}$ subpopulations from RagF5 control was analyzed by real time RT-PCR (Figure 3d). We observed a 3.2fold increase of $A 1$ expression in positively selected, $V \beta 11^{\text {high }}$ thymocytes, whereas in those cells $\mathrm{Bcl}-\mathrm{xl}$ expression was lower and expression of $\mathrm{Bcl}-2$ and $\mathrm{Mcl}-1$ only moderately increased when compared to their expression in $\mathrm{V} \beta 11^{\text {low }}$ thymocytes. These results show that $A 1$ expression is indeed upregulated in positively selected thymocytes and suggest that a fourfold increase of A1 expression in DP thymocytes is sufficient to support extended survival and therefore accumulation of non (or not yet) positively selected thymocytes.

In RagF5 Tg model, every DP thymocyte expresses the unique combination of $\mathrm{V} \alpha 4$ and $V \beta 11$ chains that forms the $F 5$ TCR. Therefore, positive selection in those mice is solely controled by self ligand- $\mathrm{H} 2 \mathrm{D}^{\mathrm{b}}$ complexes availability. We therefore speculate that if $\mathrm{A} 1$ allows survival of nonpositively selected DP thymocytes, then delivering an excess of positive selecting peptide should permit positive selection of $\mathrm{V} \beta 11^{\text {low }}$ accumulated cells. The NP34 peptide, an antagonist peptide for F5 TCR, was previously shown, in fetal organ thymic

Figure 3 A1 overexpression increases DP thymocytes survival. (a) FACSsorted DP cells from 6-week-old RagF5 (white) and RagF5-A1(black) Tg mice were cultured in medium alone (circle) or in the presence of NP68 peptide (10 $\mu \mathrm{M}$, square) or dexamethasone (10 nM, triangle). Percentage of viable cells was measured at the indicated time as described in Materials and methods. Results are expressed as the mean + S.E.M. of three independent experiments. (b) 6-week-old RagF5 (white) and RagF5-A1 (black) were i.p. injected with BrdU (130 $\mu \mathrm{g} / \mathrm{g}$ of body mass), and thymocytes were analyzed $5 \mathrm{~h}$ after injection for the incorporation of BrdU as described in Materials and methods. These results are the mean of two independent experiments. (c) Expression of $V \beta 11$ on DP thymocytes from 6-week-old RagF5 (broken line) and RagF5-A1 (solid line) Tg mice was analyzed by flow cytometry. The histograms shown are representative of at least 20 individual mice. (d) FACS-sorted DP V $\beta 11^{\text {lo }}$ and $V \beta 11^{\text {hi }}$ cells from 6-week-old RagF5 were analyzed for A1, Bcl-xl, Bcl-2 and Mcl-1 mRNA content by real-time RT-PCR as described in Materials and methods. The fold increase of $\mathrm{A} 1, \mathrm{Bcl}-\mathrm{xl}, \mathrm{Bcl}-2$ and $\mathrm{Mcl}-1$ expression between DP V $\beta 11^{\mathrm{lo}}$ and DP $\mathrm{V} \beta 11^{\mathrm{hi}}$ is shown. Results are expressed as the mean \pm S.E.M. of three independent experiments 
culture, to induce positive but not negative selection of F5 DP thymocytes, and to inhibit negative selection induced by the antigenic NP68 peptide. ${ }^{22,23}$ We therefore tested whether injection of NP34 would allow positive selection of DP cells that have accumulated in RagF5-A1 mice, by following upregulation of $\mathrm{V} \beta 11$ expression on DP population. RagF5A1 and RagF5 control mice were injected with 50 nM of NP34 antagonist peptide. After $24 \mathrm{~h}$ thymuses were collected and V $\beta 11$ expression on DP cells was analyzed by flow cytometry. As shown in Figure 4a, NP34 does not modify $\mathrm{V} \beta 11$ a
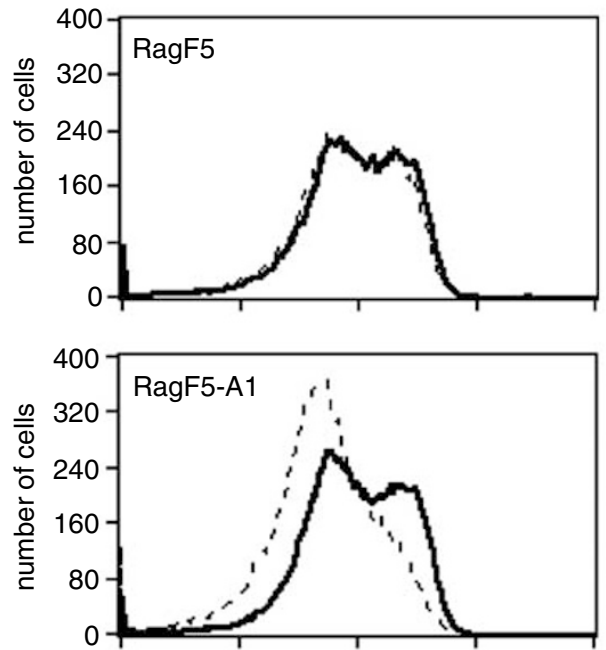

b

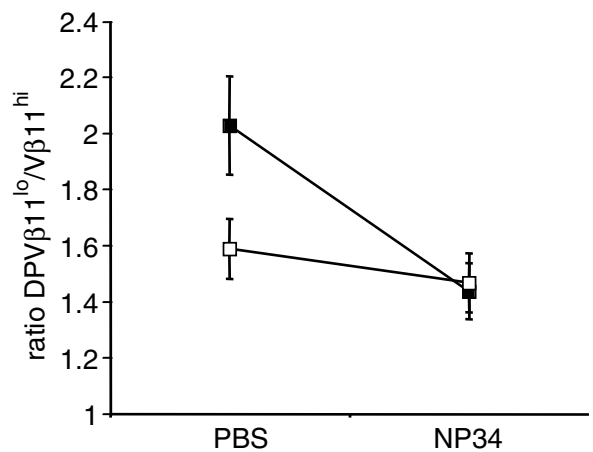

C

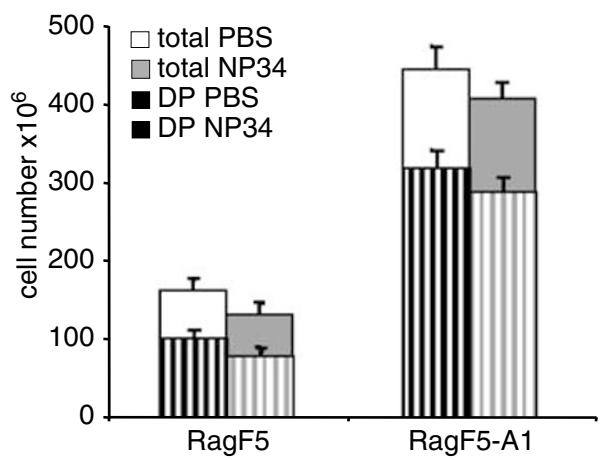

d
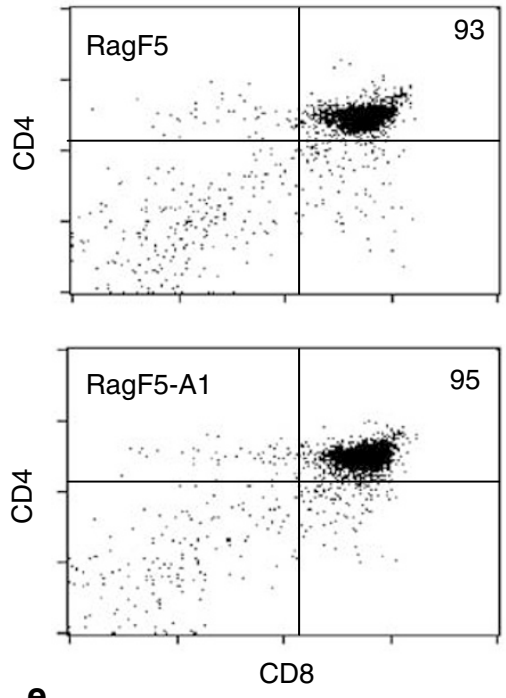

e

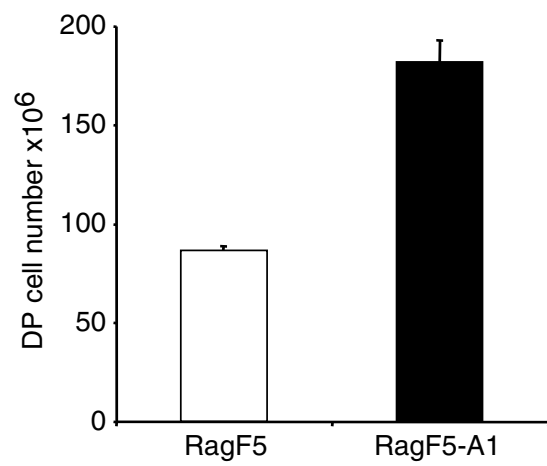

Figure 4 A1 overexpression permits the accumulation of nonpositively-selected DP thymocytes. (a, $\mathbf{b}$ and $\mathbf{c}$ ), 6-week-old RagF5 and RagF5-A1 Tg mice were i.p. injected with PBS or $50 \mathrm{nmol}$ of NP34 peptide. (a) $24 \mathrm{~h}$ later expression of V $\beta 11$ on DP thymocytes from mice treated with PBS (broken line) or NP34 peptide (solid line) was analyzed by flow cytometry. (b) Ratio of DPV $\beta 11^{10} / \mathrm{N} \beta 11^{\text {hi }}$ cells from RagF5 (white) and RagF5-A1 (black) Tg mice treated with PBS or NP34 peptide were calculated. Results are expressed as the mean \pm S.E.M. of three independent experiments. (c) Total thymocyte numbers and DP cell numbers from RagF5 and RagF5A1 Tg mice treated with PBS or NP34 were evaluated. Results are expressed as the mean +S.E.M. of three independent experiments. (d and e) 10-14-week-old B10$\mathrm{Q}-\mathrm{H} 2^{q} / \mathrm{SgJ}$ mice were lethally irradiated and reconstituted with bone-marrow cells from RagF5 or RagF5-A1 $\mathrm{Tg}$ mice. (d) 4 weeks after bone-marrow transfer, thymocytes from recipient mice were analyzed by flow cytometry for CD4 and CD8 expression. The percentage of DP cells is shown in each dot-plot. (e) 4 weeks after bone-marrow transfer, DP cell numbers were counted in recipient mice. Mean \pm S.E.M. data from five mice are shown 
expression profile in RagF5 control mice and $\mathrm{V} \beta 11^{\mathrm{lo}} / \mathrm{V} \beta 11^{\text {hi }}$ ratio is similar to the ratio observed in noninjected mice (phosphate-buffer saline (PBS)). Interestingly, injection of RagF5-A1 with NP34 peptide, increases the percentage of $\mathrm{V} \beta 11^{\text {hi }}$ expressing cells and reduces $\mathrm{V} \beta 11^{\mathrm{lo}} / \mathrm{V} \beta 11^{\mathrm{hi}}$ ratio to the level observed in RagF5 control mice (Figure $4 b$ ). Of note that immunization with NP34 peptide only marginally affects DP cell numbers (Figure $4 \mathrm{~b}$ ), indicating that upregulation of $\mathrm{V} \beta 11$ expression is not due to preferential elimination of $\mathrm{V} \beta 11^{\mathrm{lo}} \mathrm{DP}$ thymocytes following NP34 treatment.

To confirm that $A 1$ overexpression allows DP survival in the absence of positive selection, we have tested whether A1 overexpression would allow an extended survival of DP thymocytes in mice presenting a nonselecting $\mathrm{MHC}$ haplotype. The H2-q MHC haplotype is nonselecting for the F5 TCR and it as been demonstrated that $\mathrm{T}$ cell development is blocked at the DP stage in RagF5/H2 ${ }^{\mathrm{q} / \mathrm{q}}$ mice. ${ }^{9}$ We therefore analyzed thymic reconstitution from RagF5-A1 or RagF5 progenitors in $\mathrm{H}_{2}{ }^{\mathrm{q} / \mathrm{q}}$ recipient hosts. Bone marrow cells from either RagF5-A1 and RagF5 control mice were injected into irradiated B10-Q- $2^{9} / \mathrm{SgJ}$ mice and thymic reconstitution was analyzed 4 weeks after cell transfer. In agreement with previous data, ${ }^{9,24}$ we observed that thymocyte development from RagF5 progenitors is blocked at the DP stage (Figure 4d). Overexpression of A1 does not allow further T-cell development but the total DP cell number is increased by two-fold (Figure 4e), indicating that A1 overexpression allows prolonged survival of $\mathrm{DP}$ in a nonselecting $\mathrm{MHC}$ backroung.

\section{A1 overexpression does not affect negative selection of DP thymocytes}

Exogenous administration of the NP68 peptide of influenza nucleoprotein was previously demonstrated to mimic negative selection and to cause clonal depletion in RagF5 mice. ${ }^{25}$ RagF5-A1 and RagF5 control mice were therefore injected with NP68 antigenic peptide to test the effect of A1 overexpression on negative selection. As expected we observed that NP68 peptide induces a strong reduction of DP cell numbers in RagF5 control mice, with a total disappearance of $\mathrm{V} \beta 11^{\text {hi }}$ expressing cells (Figure $5 \mathrm{a}$ and $\mathrm{b}$ ). Similar results were obtained following injection of RagF5-A1 mice indicating that A1 overexpression in DP thymocyte does not inhibit negative selection.

Altogether those results suggest that $A 1$ overexpression in DP cells would favor their survival in the absence of positive selecting signals but would not interfere with death signals involved in negative selection.

\section{Discussion}

T-cell development is characterized by the production of a large number of immature thymocytes that undergo several steps of selection, initiated by either pre-TCR or TCR signals. We and others recently identified $A 1$ as a gene regulated by pre-TCR and TCR in thymocytes ${ }^{12}$ or mature CD4 and CD8T cells ${ }^{11}$ respectively. A1 consists of three highly homologous, separately encoded isoforms, A1a, A1b and A1d. A1d and $\mathrm{A} 1 \mathrm{~b}$ are the major isoforms expressed in thymocytes ${ }^{12}$ (data a
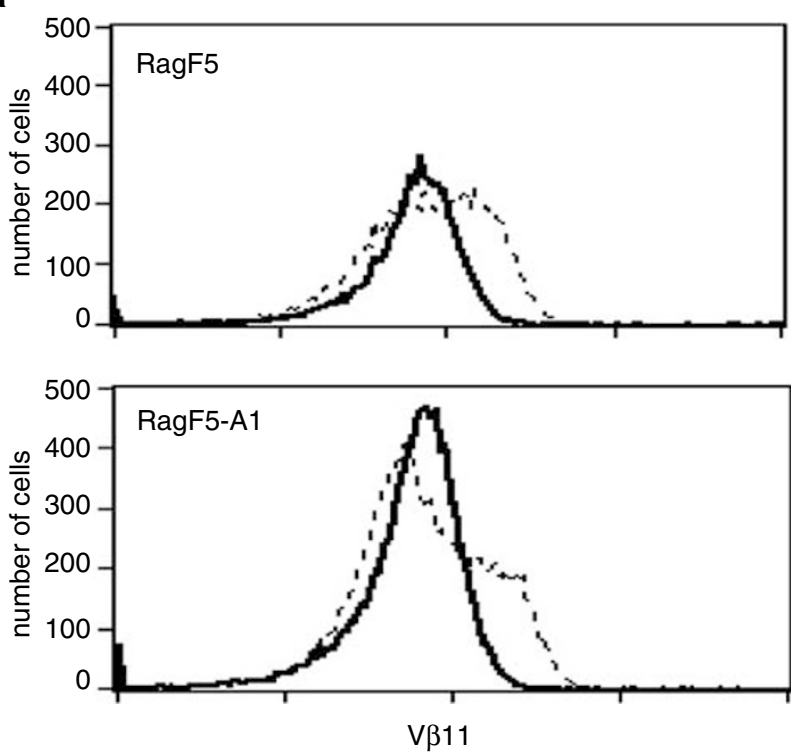

b

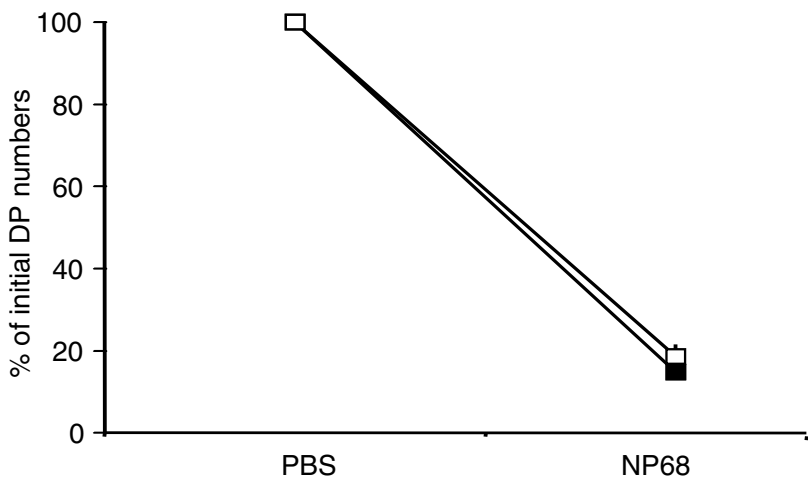

Figure 5 A1 overexpression does not affect negative selection of DP thymocytes. (a and b) 6-week-old RagF5 and RagF5-A1 $\mathrm{Tg}$ mice were i.p. injected with PBS or $50 \mathrm{nmol}$ of NP68 peptide. (a), $24 \mathrm{~h}$ after injection expression of V $\beta 11$ on DP thymocytes from mice treated with PBS (broken line) or NP68 peptide (solid line) was analyzed by flow cytometry. (b) Percentage of initial DP cell numbers from RagF5 (white) and RagF5-A1 (black) Tg mice treated with PBS or NP34 peptide were calculated. Results are expressed as the mean \pm S.E.M. of three independent experiments

not shown) and resting mature CD8T cells. ${ }^{11}$ Following antigenic stimulation of naive CD8T cells the three isoforms are upregulated with a preferential increase of $\mathrm{A} 1 \mathrm{a}$ and $\mathrm{A} 1 \mathrm{~d} .{ }^{11}$ C57BL/6 mice that overexpress the $\mathrm{A} 1$-a isoform under the control of the distal lck promoter show a marginal increased cellularity in lymphoid organs, with an average of 1.4 times more cells in the thymus. ${ }^{26}$ We observed similar results in C57BL/10 mice overexpresssing A1-a under the control of the human CD2 promoter (data not shown). Restricting TCR repertoire to a unique TCR by generating mice overexpressing A1-a and F5 TCR on the Rag-1 ${ }^{-/-}$background (RagF5A1) exacerbates thymic phenotype of A1 transgenic mice. Indeed, we observed an increased thymic cellularity in RagF5A1 mice with almost three times more thymocytes in 6-weekold mice when compared with control RagF5 mice. However, we did not observe increased cell numbers in either lymph 
nodes or spleens from RagF5-A1 mice, an observation that could be explained by the very low expression of the A1-a transgene in mature $T$ cells.

We showed that increased thymocyte number is associated with stronger accumulation of DP cells that are characterized by a higher resistance to apoptotic stimuli. Accumulation of DP cells in RagF5-A1mice is associated with a higher representation of $\mathrm{V} \beta 11^{\text {low }}$ expressing cells. These results support the hypothesis that overexpression of A1-a allows the survival of DP cells that would normally die as a result of the failure to receive positive selection signal, that is successful interaction of the TCR with self-peptide/MHC complex. This hypothesis is reinforced by our demonstration that $A 1$ overexpression promotes the accumulation of DP thymocytes even in the context of a nonselecting $\mathrm{MHC}$.

In mice expressing a unique TCR, the number of cells that will be positively selected is solely controled by the availability of endogenous self ligand presented by selecting $\mathrm{MHC}$ molecule. We showed here that exogenous administration of the NP34 antagonist peptide, a peptide which induces positive but not negative selection, restores normal $\mathrm{V} \beta 11^{\text {low }}$ $\mathrm{V} \beta 11^{\text {high }}$ ratio, demonstrating that indeed overexpression of A1 allows accumulation of DP cells awaiting for positive selection. Supporting the idea that $A 1$ may be a key protein for DP thymocyte survival, we observed that its expression is increased in positively selected thymocytes (TCR ${ }^{\text {high }}$ ) from RagF5 mice. Of note that $\mathrm{Bcl}-2$ and $\mathrm{Mcl}-1$ expression are only modestly increased and $\mathrm{Bcl}-\mathrm{xl}$ expression is downregulated between $\mathrm{V} \beta 11^{\text {low }}$ and $\mathrm{V} \beta 11^{\text {high }}$ stages.

In order to support our data it would be interesting to examine thymocyte development in A1 deficient mice. Inactivation of the $\mathrm{A} 1 \mathrm{a}$ isoform has been realized by gene targeting in mice and was reported to have only a minor impact on hematopoiesis with no apparent effect on T-cell survival. ${ }^{27}$ However, A1a isoform is poorly express in both thymocytes and mature T cells. ${ }^{11,12}$ More recently, Oberdoerffer et al. ${ }^{28}$ developped a Cre/loxP-based approach to allow for the conditional deletion of the three $\mathrm{A} 1$ isoforms by RNAi in vivo. Using that system the authors reduced A1 expression by seven-fold in thymocytes. They did not see any significant effect on thymocyte development, suggesting that a low level of $A 1$ is sufficient for T-cell development in wild-type mice or that A1 might not be essential for T-cell development. In the absence of an approach that would permit a complete and simultaneous extinction of all A1 isorforms it remain difficult to definitively address the function of $\mathrm{A} 1$ in T-cell development.

We also demonstrated in this study that overexpression of A1 in DP thymocytes does not affect negative selection in vivo. Therefore, the effect of $\mathrm{A} 1$ on $\mathrm{T}$-cell development can be clearly differentiated from that of $\mathrm{Bcl}-2$. Indeed using the RagF5 transgenic model, Williams et al., ${ }^{9}$ demonstrated that $\mathrm{Bcl}-2$ overexpression increases the total number of thymocytes by about two-fold, but at the difference from A1, Bcl-2 overexpression in RagF5 mice strongly enhances SP CD8 thymocyte population. Such an effect of Bcl-2 on a SP population can be explained by the demonstration that $\mathrm{Bcl}-2$ but not $A 1$ overexpression protects thymocytes from antigen induced negative selection. The effect of $A 1$ on DP cell survival may also be distinct from that of $\mathrm{Bcl}-\mathrm{xl}$. First the expression profile of these two genes is clearly different, $\mathrm{Bcl}-\mathrm{xl}$ being the major antiapoptotic gene expressed in DP thymocytes, however, we confirmed here that its expression is downregulated during positive selection. ${ }^{29}$ Previous data demonstrated that $\mathrm{Bcl}-\mathrm{xl}$ deletion ${ }^{3}$ or overexpression ${ }^{18}$ in the lymphoid compartment clearly affects survival of immature DP thymocytes before positive selection.

Finally despite redundant antiapoptotic function of $\mathrm{A} 1, \mathrm{Bcl}-$ 2, Bcl-xl and Mcl-1, a comparative analysis of 1/their pattern of expression in thymocyte sub-populations, 2/the effect of their deletion or overexpression on T-cell development and 3/their regulation by $\mathrm{T}$-cell survival signals, suggests that these four proteins may control different steps of T-cell development. We demonstrated here that $A 1$ expression is upregulated at both $\beta$ and positive selection steps and that $A 1$ is capable of substituting for positively selecting signals, suggesting a specific function of A1 in pre-TCR and TCR-mediated survival signals during T-cell development. In contrast $\mathrm{Mcl}-1$ and $\mathrm{Bcl}-$ 2 , two cytokine-regulated proteins, ${ }^{10,11}$ would be essential to control cytokine-dependent steps of thymocyte survival. Indeed Mcl-1 was demonstrated to be essential for survival of DN2 cells that undergo TCR rearrangement and are highly dependent on cytokines for their survival. ${ }^{10}$ Similarly Bcl-2 overexpression was demonstrated to rescue T-cell development in IL-7 receptor deficient mice but not in $\mathrm{Rag}^{-1-}$ mice. ${ }^{30}$ IL-7 is also a critical cytokine for survival of newly positively selected DP thymocytes and their differentiation into functionally SP $\mathrm{T}$ cells. At this stage IL-7 was demonstrated to upregulate $\mathrm{Bcl}-2$ expression and therefore to provide survival signal to positively selected DP thymocytes. ${ }^{31}$ We and others demonstrated that $\mathrm{Bcl}-\mathrm{xl}$ is regulated by both TCR or cytokine receptor signals in mature $\mathrm{T}$ cells, ${ }^{11,32}$ however, its specific pattern of expression in thymocytes after the pre-TCR checkpoint during the time of TCRA rearrangement may reflect its regulation by other critical signals such as Wntfrizzled mediated signals. ${ }^{33,34}$

In conclusion, we propose that $A 1$ expression may be crucial for the survival of DP thymocytes. Survival of DP thymocytes would be sequentially controlled, first by Bcl-xl during their migration from the cortex to the medulla then by TCR-mediated positive selection signals that would primarily upregulate A1. A1 upregulation would allow survival of newly selected DP cells, that will re-express IL-7R $\alpha$ chain and then become sensitive to IL-7 which will provide survival signal by upregulating $\mathrm{Bcl}-2$ (and/or $\mathrm{Mcl}-1$ ) expression, but also signals that are required for their differentiation into functionally

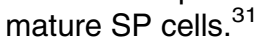

\section{Materials and Methods}

\section{Real time PCR}

Thymocytes subpopulations were FACS-sorted according to CD4, CD8, CD117, CD44 and CD25 expression as described by Ceredig et al..$^{35}$ Total RNA was isolated by using NucleoSpin ${ }^{\circledR}$ RNA II kit (Macherey-Nagel). After DNase treatment (Pharmacia Biotech, Amersham), cDNA templates were obtained from $1 \mu \mathrm{g}$ total RNA using Superscript II-RT kit (Invitrogen) with random hexamer primers (Promega). Detection of antiapoptotic genes from $\mathrm{Bcl}-2$ family was made using following primer pairs: A1F: $5^{\prime}$-gattgccetggatgtatgtgcttac- $3^{\prime}$ and A1R: $5^{\prime}$-agccatcttcccaacctccattc-3'; Bcl-xIF: $5^{\prime}$-agcaggtagtgaatgaactcttcg-3' Bcl-xIR: $5^{\prime}$-ccatccaacttgcaatccgactc-3'; 
Bcl-2F: $5^{\prime}$-tacagcattgcggaggaagtagac-3' and Bcl-2R: $5^{\prime}$ - ttagttacaccgaa cacttgattctgg-3'; Mcl-1F: $5^{\prime}$-gctccggaaactggacatta-3' and Mcl-1R: $5^{\prime}$ cccagtttgttacgccatct- $3^{\prime}$. HPRT and Ubiquitine transcripts were detected using HPRTF: 5'-tcattatgccgaggatttgga-3' and HPRTR: 5'-cagagggcca caatgtgatg- $3^{\prime}$, UbiquitineF: $5^{\prime}$-aagaattcagatcggatgacacact- $3^{\prime}$ and UbiquitineR: $5^{\prime}$-gccacttggaggttgacactt- $3^{\prime}$. PCR efficiencies were comprised between 1.88 and 2.02. The PCR were performed with the Platinium SYBR Green qPCR SuperMix UDG (Invitrogen) on an Applied Biosystems GeneAmp 7600 thermocycler. Each sample was run in triplicate and data were analyzed in order to determine the relative gene expression of each antiapoptotic genes. Gene expression was normalized using geometric mean of housekeeping genes expression ${ }^{36}$ and relative gene expression was calculated as previously described. ${ }^{37}$

\section{Mice}

In this study, we used C57BL/10 mice, B10-Q-H2 $/ \mathrm{SgJ}$, RagF5 Tg mice that express a TCR recognizing an $\mathrm{H} 2-\mathrm{D}^{\mathrm{b}}$-restricted $\mathrm{A} / \mathrm{NT} / 60 / 68$ influenza virus NP epitope (NP68) and RagF5-A1 Tg mice that over-expressed a Nterminally flag-tagged version of the $A 1-a$ gene $(f A 1)$ under the control of the human $\mathrm{CD} 2$ minigene. ${ }^{38}$ The retroviral vector containing the $\mathrm{fA} 1 \mathrm{cDNA}$ (pEYZ/FmA1) has been described previously. ${ }^{39} \mathrm{~A}$ Xna I/Kpn2 I fragment containing the $\mathrm{fA} 1$, an internal ribosomal entry site (IRES) and a chimeric selection marker (eYFP/Zeocine) was inserted into the Sma I site of the human CD2 minigene cassette. The linearized hCD2-fA1-IRES-YZ cassette was microinjected into fertilized eggs from B6D2 mice. $\mathrm{Tg}$ founder mice were identified by PCR on genomic DNA with the following primers 5Flag: $5^{\prime}$-agcttggatccgaattcagcatggactacaaggacgacgat gacaaggc- $3^{\prime}$ and $3 \mathrm{mA1}$ : 5'-cgcggatccgtgttacttgaggagaaagag- $3^{\prime}$. A1 Tg mice were backcrossed to $\mathrm{C} 57 \mathrm{BL} / 10$ mice for more than 10 generations and then bred to RagF5 Tg mice. The genotyping of mice for the presence of F5 TCR, A1 transgene and the presence of Rag-1 genes was done by $\mathrm{PCR}$, dot blots or flow cytometry. All mice, except B10-Q-H2 ${ }^{q} / \mathrm{SgJ}$ mice that were obtained from The Jackson laboratory (Bar Harbor, ME), were bred at the 'Plateau de Biologie Expérimentale de la Souris' under specific pathogen-free conditions and used at an age of day 1-15 weeks. For bone-marrow transfers, 10-14-week-old B10-Q- $\mathrm{H}^{\mathrm{q}} / \mathrm{SgJ}$ mice were lethally irradiated with $\gamma$-irradiation ( $9 \mathrm{~Gy}$ ) $5 \mathrm{~h}$ before reconstitution with $5 \times 10^{6}$ bone-marrow cells from donor mice. Recipient mice were analyzed 4 weeks after transfer.

\section{Peptide treatment of RagF5 and RagF5-A1 Tg mice}

Mice (5 weeks old) were injected intraperitoneally with PBS or peptides diluted in PBS. The following peptides $(50 \mathrm{nmol})$ were injected in RagF5 and RagF5-A1 transgenic mice: NP68 from the nucleoprotein of influenza virus A/NT/60/68 (NP366-374): ASNENMDAM and NP34 from the nucleoprotein of influenza virus A/PR/8/34 (NP366-374): ASNENMETM (Dr D Fischeux, Institut de Biologie et Biochimie des Protéines, Lyon, France).

\section{Cell culture}

DP thymocytes from 6 weeks-old mice were FACS-sorted according to CD4 and CD8 expression using FACSvantage SE option Diva (Becton Dickinson, Le Pont de Claix, France). Purity of DP isolated thymocytes was greater than $99 \%$. FACS-sorted DP thymocytes were cultured at $1210^{6} \mathrm{cells} / \mathrm{ml}$ in RPMl 1640 medium supplemented with $10 \%$ FCS, $2 \mathrm{mM}$ L-glutamine, $40 \mu \mathrm{g} / \mathrm{ml}$ gentamycin (Invitrogen Life Technologies, Cergy Pontoise, France) and $50 \mu \mathrm{g} / \mathrm{ml}$ 2-ME (Sigma-Aldrich) alone or in the presence of $10 \mu \mathrm{M}$ of NP68 (366-374) peptide or $10 \mathrm{nM}$ of dexamethasone (Sigma-Aldrich).

\section{Cell staining and flow cytometry}

The following antibodies for flow cytometry were obtained from $\mathrm{BD}$ Pharmingen: anti-CD $8 \alpha-\mathrm{FITC}$, anti-CD $8 \alpha-\mathrm{PE}$, anti-Vb11-PE, anti-CD25PE, anti-CD4-PerCp-Cya5.5, anti-CD8 $\alpha$-PerCp-Cya5.5 and anti-CD44APC. Pentamere anti-TCR F5 was purshased from Proimmune. For in vivo 5-bromo-2'-deoxyuridine (BrdU) labeling experiments, mice were injected with BrdU (BD Pharmingen) (130 $\mu \mathrm{g} / \mathrm{g}$ of body mass, i.p.). At $5 \mathrm{~h}$ after injection, thymocytes were isolated and surface stained with antiCD25-PE, anti-CD4-PerCp-Cya5.5, anti-CD8 $\alpha$-PerCp-Cya5.5 and antiCD44-APC or anti-CD8 $\alpha$-PE and anti-CD4-PerCp-Cya5.5. The cells were the fixed and intracellularly stained with anti-BrdU-FITC using the BrdU flow kit from BD Pharmingen.

For the in vitro survival assay, apoptosis of FACS-sorted DP thymocytes was evaluated by annexin $\mathrm{V}$ staining (annexin V-FITC, BD Pharmingen).

\section{Acknowledgements}

We would like to thank Dr Jacqueline Marvel for helpful discussions, Antoine Marçais for his help in adoptive transfer experiments and Dr. Janet Maryanski for critical reading of the manuscript. This work is supported by institutional grants from INSERM and UCB Lyon I, and additional support from the Association pour la Recherche sur le Cancer, The Ligue contre le cancer (comité du rhône), the Région Rhône-Alpes and Cancéropole National (NBB). C Verschelde is supported by a fellowship from The Association pour la Recherche sur le Cancer. I Berberich was supported by the Wilhem Sander-Stiftung (1999.107.2).

\section{References}

1. Fehling $\mathrm{HJ}$ and von Boehmer $\mathrm{H}$ (1997) Early alpha beta T cell development in the thymus of normal and genetically altered mice. Curr. Opin. Immunol. 9: 263-275

2. Huesmann M, Scott B, Kisielow P and von Boehmer H (1991) Kinetics and efficacy of positive selection in the thymus of normal and $\mathrm{T}$ cell receptor transgenic mice. Cell 66: 533-540

3. Ma A, Pena JC, Chang B, Margosian E, Davidson L, Alt FW and Thompson CB (1995) Bclx regulates the survival of double-positive thymocytes. Proc. Natl. Acad. Sci. USA 92: 4763-4767

4. Linette GP, Grusby MJ, Hedrick SM, Hansen TH, Glimcher LH and Korsmeyer SJ (1994) Bcl-2 is upregulated at the CD4+ CD8+ stage during positive selection and promotes thymocyte differentiation at several control points. Immunity 1: 197-205

5. Veis DJ, Sentman CL, Bach EA and Korsmeyer SJ (1993) Expression of the $\mathrm{Bcl}-2$ protein in murine and human thymocytes and in peripheral $\mathrm{T}$ lymphocytes. J. Immunol. 151: 2546-2554

6. Gratiot-Deans J, Merino R, Nunez G and Turka LA (1994) Bcl-2 expression during T-cell development: early loss and late return occur at specific stages of commitment to differentiation and survival. Proc. Natl. Acad. Sci. USA 91: 10685-10689

7. Strasser A, Harris AW, von Boehmer H and Cory S (1994) Positive and negative selection of $\mathrm{T}$ cells in T-cell receptor transgenic mice expressing a bcl2 transgene. Proc. Natl. Acad. Sci. USA 91: 1376-1380

8. Tao W, Teh SJ, Melhado I, Jirik F, Korsmeyer SJ and Teh HS (1994) The T cell receptor repertoire of CD4-8+ thymocytes is altered by overexpression of the BCL-2 protooncogene in the thymus. J. Exp. Med. 179: 145-153

9. Williams O, Norton T, Halligey M, Kioussis D and Brady HJ (1998) The action of Bax and Bcl-2 on T cell selection. J. Exp. Med. 188: 1125-1133 
10. Opferman JT, Letai A, Beard C, Sorcinelli MD, Ong CC and Korsmeyer S (2003) Development and maintenance of $B$ and $T$ lymphocytes requires antiapoptotic MCL-1. Nature 426: 671-676

11. Verschelde C, Walzer T, Galia P, Biemont MC, Quemeneur L, Revillard JP, Marvel $\mathrm{J}$ and Bonnefoy-Berard N (2003) A1/Bfl-1 expression is restricted to TCR engagement in T lymphocytes. Cell Death Differ. 10: 1059-1067

12. Mandal M, Borowski C, Palomero T, Ferrando AA, Oberdoerffer P, Meng F, Oberdoerffer P, Meng F, Ruiz-Vela A, Ciofani M, Zuniga-Pflucker JC, Screpanti I, Look AT, Korsmeyer SJ, Rajewsky K, von Boehmer H and Aifantis A (2005) The BCL2A1 gene as a pre-T cell receptor-induced regulator of thymocyte survival. J. Exp. Med. 201: 603-614

13. Palmer MS, Bentley A, Gould K and Townsend AR (1989) The T cell receptor from an influenza-A specific murine CTL clone. Nucleic Acids Res. 17: 2353

14. Mamalaki C, Elliott J, Norton T, Yannoutsos N, Townsend AR, Chandler P, Simpson $E$ and Kioussis D (1993) Positive and negative selection in transgenic mice expressing a T-cell receptor specific for influenza nucleoprotein and endogenous superantigen. Dev. Immunol. 3: 159-174

15. Sentman CL, Shutter JR, Hockenbery D, Kanagawa $O$ and Korsmeyer SJ (1991) bcl-2 inhibits multiple forms of apoptosis but not negative selection in thymocytes. Cell 67: 879-888

16. Strasser A, Harris AW and Cory S (1991) bcl-2 transgene inhibits T cell death and perturbs thymic self- censorship. Cell 67: 889-899

17. Chao DT, Linette GP, Boise LH, White LS, Thompson CB and Korsmeyer SJ (1995) Bcl-XL and Bcl-2 repress a common pathway of cell death. J. Exp. Med. 182: $821-828$

18. Grillot DA, Merino R and Nunez G (1995) Bcl-XL displays restricted distribution during $T$ cell development and inhibits multiple forms of apoptosis but not clonal deletion in transgenic mice. J. Exp. Med. 182: 1973-1983

19. von Boehmer $\mathrm{H}$ (2004) Selection of the T-cell repertoire: receptor-controlled checkpoints in T-cell development. Adv. Immunol. 84: 201-238

20. Ohashi PS, Pircher H, Burki K, Zinkernagel RM and Hengartner H (1990) Distinct sequence of negative or positive selection implied by thymocyte T-cell receptor densities. Nature 346: 861-863

21. Haks MC, Pepin E, van den Brakel JH, Smeele SA, Belkowski SM, Kessels HW, Krimpenfort P and Kruisbeek AM (2002) Contributions of the T cell receptor-associated CD3gamma-ITAM to thymocyte selection. J. Exp. Med. 196: 1-13

22. Williams O, Mok CL, Norton T, Harker N, Kioussis D and Brady HJ (2001) Elevated Bcl-2 is not a causal event in the positive selection of T cells. Eur. J. Immunol. 31: 1876-1882

23. Smyth LA, Williams O, Huby RD, Norton T, Acuto O, Ley SC and Kioussis D (1998) Altered peptide ligands induce quantitatively but not qualitatively different intracellular signals in primary thymocytes. Proc. Natl. Acad. Sci. USA 95: 8193-8198

24. Tanaka Y, Williams O, Tarazona R, Wack A, Norton T and Kioussis D (1997) In vitro positive selection of alpha beta TCR transgenic thymocytes by a conditionally immortalized cortical epithelial clone. Int. Immunol. 9: 381-393
25. Mamalaki C, Norton T, Tanaka Y, Townsend AR, Chandler P, Simpson E and Kioussis D (1992) Thymic depletion and peripheral activation of class I major histocompatibility complex-restricted $T$ cells by soluble peptide in T-cell receptor transgenic mice. Proc. Natl. Acad. Sci. USA 89: 11342-11346

26. Gonzalez J, Orlofsky A and Prystowsky MB (2003) A1 is a growth-permissive antiapoptotic factor mediating postactivation survival in T cells. Blood 101: 2679-2685

27. Hamasaki A, Sendo F, Nakayama K, Ishida N, Negishi I and Hatakeyama S (1998) Accelerated neutrophil apoptosis in mice lacking A1-a, a subtype of the bcl-2-related A1 gene. J. Exp. Med. 188: 1985-1992

28. Oberdoerffer P, Kanellopoulou C, Heissmeyer V, Paeper C, Borowski C, Aifantis I, Rao A and Rajewsky K (2005) Efficiency of RNA interference in the mouse hematopoietic system varies between cell types and developmental stages. Mol. Cell Biol. 25: 3896-3905

29. Chao DT and Korsmeyer SJ (1997) BCL-XL-regulated apoptosis in T cell development. Int. Immunol. 9: 1375-1384

30. Maraskovsky E, O'Reilly LA, Teepe M, Corcoran LM, Peschon JJ and Strasser A (1997) Bcl-2 can rescue T lymphocyte development in interleukin-7 receptordeficient mice but not in mutant rag-1-/- mice. Cell 89: 1011-1019

31. Yu Q, Erman B, Bhandoola A, Sharrow SO and Singer A (2003) In vitro evidence that cytokine receptor signals are required for differentiation of double positive thymocytes into functionally mature CD8+ T cells. J. Exp. Med. 197: $475-487$

32. Boise LH, Minn AJ, Noel PJ, June CH, Accavitti MA, Lindsten T and Thompson CB (1995) CD28 costimulation can promote T cell survival by enhancing the expression of Bcl-XL. Immunity 3: 87-98

33. Ioannidis V, Beermann F, Clevers $\mathrm{H}$ and Held W (2001) The beta-catenin-TCF1 pathway ensures $\mathrm{CD} 4(+) \mathrm{CD} 8(+)$ thymocyte survival. Nat. Immunol. 2: 691-697

34. Wu L and Strasser A (2001) 'Decisions, decisions.' beta-catenin-mediated activation of TCF-1 and Lef-1 influences the fate of developing T cells. Nat. Immunol. 2: 823-824

35. Ceredig R and Rolink T (2002) A positive look at double-negative thymocytes. Nat. Rev. Immunol. 2: 888-897

36. Pfaffl MW, Tichopad A, Prgomet C and Neuvians TP (2004) Determination of stable housekeeping genes, differentially regulated target genes and sample integrity: BestKeeper - Excel-based tool using pair-wise correlations. Biotechnol. Lett. 26: 509-515

37. Pfaffl MW (2001) A new mathematical model for relative quantification in realtime RT-PCR. Nucleic Acids Res. 29: e45

38. Zhumabekov T, Corbella P, Tolaini M and Kioussis D (1995) Improved version of a human CD2 minigene based vector for $T$ cell-specific expression in transgenic mice. J. Immunol. Methods 185: 133-140

39. Kuss AW, Knodel M, Berberich-Siebelt F, Lindemann D, Schimpl A and Berberich I (1999) A1 expression is stimulated by CD40 in B cells and rescues WEHI 231 cells from anti-IgM-induced cell death. Eur. J. Immunol. 29: 3077-3088 